\title{
Streptococcus suis Meningitis in a French Cooker: Case Report and Review
}

\author{
Astrid Malézieux-Picard ${ }^{1}$, Anne De Monte1, Nicolas Degand ${ }^{1}$, Claire Grech'1, Charles Raffaelli1, \\ Jean Dellamonica ${ }^{1}$, Pierre-Eric Danin¹, Olivier Dauwalder², Eric Cua'1, Karine Risso' \\ ${ }^{1}$ Centre Hospitalo-Universitaire de Nice, France \\ ${ }^{2}$ Laboratoire de Bactériologie, Centre de Biologie et Pathologie Est, Hospices Civils de Lyon, France
}

\begin{abstract}
Streptococcus suis is an emerging zoonotic pathogen, increasingly reported worldwide and especially in Asia. S. suis remains rare in France but is responsible for outbreaks in Asia, causing meningitis, septicemia, endocarditis, arthritis and septic shock. Transmission of S. suis to humans may occur by close contact with sick or healthy pigs. Deafness is a common and often permanent neurological sequella of S. suis meningitis. We report here a case of S. suis meningitis in a French cooker specialized in pig meat diagnosed by $16 \mathrm{~S}$ rRNA PCR performed from Cerebrospinal fluid. J Microbiol Infect Dis 2016; 6(3): 136-139
\end{abstract}

Key words: Streptococcus suis, bacterial meningitis, hearing loss, rRNA $16 \mathrm{~S}$

\section{INTRODUCTION}

Streptoccoccus suis is a major swine pathogen responsible for important economic cost in the porcine industry worldwide. S. suis can be transmitted to humans by direct contact [1]. Human infection due to $S$. suis has become a major cause of anthropozoonosis and has been mostly reported in countries with intensive swine production, especially in Asia, due to a high density of pigs and to the consumption of uncooked or lightly cooked pig products. In Europe, cases involving this pathogen remain rare. To our knowledge, only seven cases have been reported so far in France. On the basis of polysaccharidic capsular antigenic differences, 35 serotypes of $S$. suis have been described. Serotype 2 is the most frequent and the most virulent in both humans and pigs [1]. S. suis can cause a wide variety of diseases in humans including meningitis, septicemia, endocarditis, pneumonia and arthritis. Permanent hearing loss or vestibular dysfunctions are common sequelae of $S$. suis infections, especially in patients with meningitis. We report here a case of $S$. suis meningitis in a French cooker diagnosed by $16 \mathrm{~S}$ rRNA PCR performed from Cerebrospinal fluid.

\section{CASE}

A 45-year-old man, with an unremarkable medical history, was transferred into intensive care unit for hyperthermic coma. For 3 days the patient had presented with fever, severe ear pain and left gonalgia for which corticosteroids were prescribed by his general practitioner. Because of the onset of a meningeal syndrome with sudden headache, vomiting, confusion and agitation, the patient received 2 $\mathrm{g}$ of ceftriaxone and was admitted to the emergency ward of the teaching Hospital of Nice. The patient presented arthritis of the left knee and elbow, which required a broad spectrum antibiotic therapy with cefotaxime, amoxicillin and gentamicin. Cerebral CT showed a diffuse cerebral edema. Laboratory investigations revealed inflammation markers such as leucocytosis $\left(31 \times 10^{9} / \mathrm{L}\right)$ with hyperpolymorphonuclear neutrophil $\left(29 \times 10^{\%} / \mathrm{L}\right)$ and elevated C-reactive protein (CRP) $(291 \mathrm{mg} / \mathrm{L})$. Cerebrospinal fluid (CSF) analysis, performed after antibiotic administration, retrieved a purulent fluid with high cellular reaction (12800cells $/ \mu \mathrm{L}$ with $96 \%$ polymorphonuclear cells), high protein concentration (3.26 $\mathrm{g} / \mathrm{L})$, low glucose concentration (3.65 mmol/L). Glycemia was $8.66 \mathrm{mmol} / \mathrm{L}$. Gram staining of the CSF was negative. CSF culture was negative after 10 days. Bacteriological analysis of the left knee Synovial fluid showed a purulent aspect, with 4600 cells/ $\mu \mathrm{L}$ dominated by polymorphonuclear cells. Gram staining of the synovial fluid was negative. Cultures remained negative after 21 days incubation. The CSF and synovial fluids were sent for $16 \mathrm{~S}$

Correspondence: Astrid Malézieux-Picard, Service de maladies infectieuses, Hôpital Archet 1, Centre Hospitalo-Universitaire de Nice, 151 Route de Saint Antoine de Ginestière, 06200 Nice, France Email: astrid.malezieux@hotmail.fr 
rRNA PCR analysis. Briefly, CSF and synovial fluid were digested followed by automated extraction on MagNa Pure compact $\circledast$ instrument using DNA Bacteria protocol. An adapted broad-range 16S rRNA using $91 \mathrm{E}$ and 13BS primers was performed on conventional thermocycler (Biometra, Germany) as previously described [2,3]. A 440-bp DNA fragment was sequenced by Biofidal (Villeurbanne, France). Definitive identification was obtained by comparison with those available in the $\mathrm{BiBi}$ database (https:// umr5558-bibiserv.univ-lyon1.fr/lebibi/lebibi.cgi) by phylogenic analysis [4]. Whereas the 16S rRNA PCR remained negative on synovial fluid, an amplification was obtained on CSF allowing the identification of Streptococcus suis with $99 \%$ similarity with the $16 \mathrm{~S}$ rRNA gene sequence (GenBank $®$ accession number AF009477).

The patient works as a cook in a restaurant specialized in pig meat. The patient had a finger cut while working 15 days before, which is likely to have enabled the infection, which can be considered a professional disease.

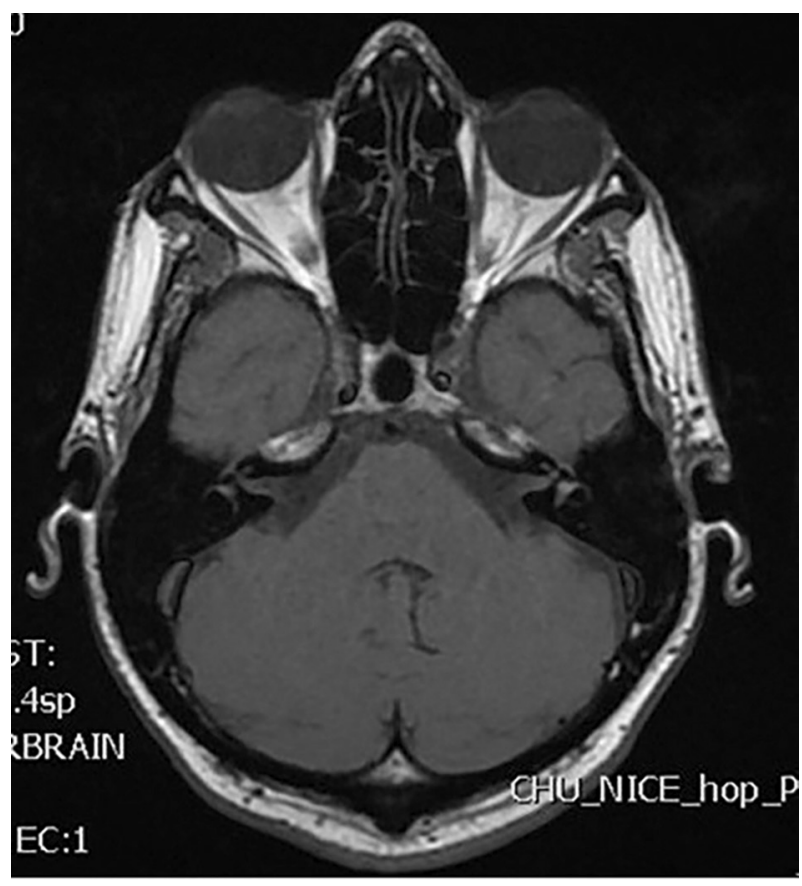

Figure 1. Axial gadolinium-enhanced T1-weighted Magnetic resonance image shows abnormal semicircular canal enhancement on the left side.

Amoxicillin and gentamicin were stopped, and cefotaxime was continued for 3 weeks in combination with rifampicin because of a suspected arthritis. Clinical examination did not reveal any valvular regurgitation and trans-thoracic echocardiogram was normal. The antibiotic therapies allowed a rapid clinical improvement of both meningitis and arthritis. However, a bilateral hearing loss was diagnosed. Magnetic resonance imaging (MRI) of the internal auditory meatus revealed abnormal bilateral enhancement of the cochlea and the labyrinthine with patchy hyperintensity in the unenhanced T1-weighted images, suggesting a hemorrhage in the cochlea (Figure 1).

Four months after the onset of the disease, loss of hearing persisted. MR imaging showed a reduced fluid signal in both cochleas. The patient underwent a right cochlear implantation followed by audiologic intensive rehabilitation in order to improve communication skills.

\section{DISCUSSION}

We report here the $8^{\text {th }}$ human case of $S$. suis infection in France. Streptococcus suis infection is recognized as a professional disease since 1995.

This pathogen, mostly retrieved in pigs, can cause severe infections in humans. Healthy pigs can carry S. suis in the upper respiratory tract, tonsils, nasal cavity and genital and digestive tract [5] In addition to pigs, $S$. suis can colonize or infect other animals, such as rodents, cats, dogs, deers, and horses [6].

Human S. suis infections are more frequent in countries where pig-rearing is common. Mean age is 47-55 years and occupational disease is higher among men (sex ratio: 3.5 to 6.5 ) [7,8]. In developed countries, the annual risk of $S$. suis meningitis among slaughterhouse workers and pig breeders has been estimated up to 3 cases per 100,000 inhabitants [9].

The incubation period of $S$. suis infections ranges from 3 hours to 14 days (median 2.2 days) [8]. Patients are generally healthy prior to infection although predisposing factors, such as splenectomy, diabetes, alcoholism, malignancy, and structural heart diseases were reported [10]. The mortality rate retrieved from published clinical case series vary from 2.6 to $19 \%$ depending on country characteristics [5]. Streptococcus suis causes a systemic infection in humans with severe organ involvement. Meningitis is the most common clinical manifestation, complicated by permanent hearing loss or vestibular dysfunction [11]. The clinical presentation of S. suis meningitis is generally similar to that of other pyogenic bacterial meningitis. The hearing loss is usually deep and bilateral, and can occur at the ini- 
tial clinical phase or a few days later. It is associated in one-half of patients with poor prognosis. Vestibular dysfunction has also been described. Less frequently, S. suis can cause acute and subacute endocarditis, acute arthritis, endophthalmitis, uveitis, spondylodiscitis and epidural abscess [12].

In humans, S. suis infection is thought to be acquired either through skin lesions or by ingestion. The presence a capsular polysaccharide (CPS) as well as other cell wall components may protect the bacteria from phagocytosis and also contribute to bacterial survival [5]. Recent results showed that $S$. suis is able to adhere and invade epithelial cells from the choroid plexus, due to suilysin (hemolysin) production. This is likely to explain the brain tropism. The toxic effect of suilysin may also contribute to an increased permeability of the blood-brain barrier. After breaching epithelial barriers, $S$. suis reaches and survives in the bloodstream, invades different organs and causes overwhelming inflammation. Inflammation of the central nervous system leads to intracranial complications, including edema, increased intracranial pressure and cerebrovascular damages [13]. Streptococcus suis is believed to enter the perilymph via the cochlear aqueduct through the lytic action of exotoxins. The infective and inflammatory processes can cause labyrinthine haemorrhage that can be seen in MRI [11].

Streptococcus suis can be isolated from CSF or blood samples with the use of standard microbiological methods. Gram staining reveals Grampositive cocci or ovoid-shaped, frequently in pairs, but sometimes alone or in short chains [14]. The majority of strains are alpha-haemolytic on bovine blood agar plates after 24 hours of incubation at $37{ }^{\circ} \mathrm{C}$ [14]. But growth is inhibited on media containing $6.5 \% \mathrm{NaCl}$. Identification of $S$. suis can be performed by Matrix Assisted Laser Desorption Ionization - Time Of Flight mass spectrometry (MALDITOF MS) or by biochemical methods, such as API, Vitek 2 or Phoenix systems, with a negative result for Voges-Proskauer test (VP), acid production from trehalose and salicin tests and resistance to optochin [14]. S. suis is often underdiagnosed because of negative cultures due to prior antibiotic use and some authors also suggest misidentifications with several other streptococci, enterococci or even Listeria [14]. Molecular PCR-based techniques have improved the rate of detection of $S$. suis. Performed directly of CSF sample, the PCR targeting the gene encoding 16S ribosomal RNA (16S rRNA) allow bacterial detection and identification. This method is useful to detect bacteria from normally sterile flu- ids or cavities, especially in case of patients receiving antibiotic therapy prior to specimen collection, in case of cultivable bacteria, or in case of samples with low culture sensitivity such as bone and joint samples, fluids from sterile cavities. Specific-PCR based methods using a $S$. suis serotype 2 cps $2 \mathrm{~J}$ gene primers can also improve the detection of $S$. suis [15].

The Antibiotic treatment should be started without delay with the same posology and duration as for pneumococcal meningitis. Mai et al. showed in a prospective study in Vietnam that $S$. suis is susceptible to penicillin, ceftriaxone and vancomycin [7]. An absence of clinical improvement under treatment, or a clinical relapse, may be due to intracranial abscess, metastatic infection, or the development of drug resistance. In case of endocarditis, endophtalmitis, or arthritis, the recommended guidelines should be observed in terms treatment duration, monitoring, and surgical intervention. The use of dexamethasone to reduce mortality and improve the outcome remains controversial.

In conclusion, infections due to $S$. suis are rare in France, but can lead to serious damage in healthy patients who are professionally exposed to pigs (slaughterhouse workers, pig breeders, butchers, veterinarians, etc.). Preventive measures, such as the use of gloves during pig meat processing and pork cooking should prevent most of cases. In case of severe bacterial meningitis, with possible occupational exposure, S. suis infection has to be suspected. In France, this occupational disease is a notifiable disease. Molecular biology is an accurate tool to identify this pathogen and resulted helpful in this case report.

\section{Acknowledgement}

The authors declared that there is not conflict of interest.

Declaration of Conflicting Interests: The authors declare that they have no conflict of interest.

Financial Disclosure: No financial support was received.

\section{REFERENCES}

1. Lun ZR, Wang QP, Chen XG, Li AX, Zhu XQ. Streptococcus suis: an emerging zoonotic pathogen. Lancet Infect Dis 2007; 7:201-209.

2. Relman D A. Universal bacterial $16 S$ rRNA amplification and sequencing. In: Persing DH, Smith TF, Tenover FC, White TJ, editors. Diagnostic Molecular Microbiology: Principles 
and Applications. Washington, DC: American Society of Microbiology. 1993. p 489-495.

3. Gauduchon V, Chalabreysse L, Etienne J, et al. Molecular diagnosis of infective endocarditis by PCR amplification and direct sequencing of DNA from valve tissue. J Clin Microbiol 2003; 41(2):763-766.

4. Devulder G, Perrière G, Baty F, Flandrois JP. BIBI, a bioinformatics bacterial identification tool. J Clin Microbiol 2003; 41(4):1785-1787.

5. Wertheim HF, Nghia HD, Taylor W, Schultsz C. Streptococcus suis: an emerging Human Pathogen. Clin Infect Dis 2009; 48:617-625.

6. Staats JJ, Feder I, Okwumabua O, Chengappa MM. Streptococcus suis: past and present. Vet Res Commun 1997; 21: 381-407.

7. Mai NT, Hoa NT, Nga TV, et all. Streptococcus suis: meningitis in adults in Vietnam. Clin Infect Dis 2008; 46: 659-667.

8. Yu H, Jing $H$, Chen Z, et al. Human Streptococcus suis outbreak, Sichuan, China. Emerg Infect Dis 2006; 12: 914-920.
9. Arends J P, Zanen H C. Meningitis caused by Streptococcus suis in humans. Rev Infect Dis 1988; 10:131-137.

10. Huang YT, Teng LJ, Ho SW, Hsueh PR. Streptococcus suis infection. J Microbiol Immunol Infect 2005; 38:306-313.

11. Tan JH, Yeh BI, Seet CS. Deafness due to haemorrhagic labyrinthitis and a review of relapses in Streptococcus suis meningitis. Singapore Med J 2010; 51(2):e30.

12. Choi $\mathrm{SM}$, Cho $\mathrm{BH}$, Choi $\mathrm{KH}$, et all. Meningitis caused by Streptococcus suis: case report and review of the literature. J Clin Neurol 2012; 8:79-82.

13. Fittipaldi N, Segura M, Grenier D, Gottschalk M. Virulence factors involved in the pathogenesis of the infection caused by the swine pathogen and zoonotic agent Streptococcus suis. Future Microbiol 2012; 7(2):259-279.

14. Higgins R, Gottschalk M. An update on Streptococcus suis identification. J Vet Diagn Invest 1990; 2(3):249-252.

15. Nga TV, Nghia HD, Tu le TP, et al. Real-time PCR for detection of Streptococcus suis serotype 2 in cerebrospinal fluid of human patients with meningitis. Diagn Microbiol Infect Dis 2011; 70(4):461-467. 